\title{
Produção de mudas de teca sob diferentes substratos orgânicos
}

\author{
Production of teak seedlings in different organic substrates \\ Producción de plántulas de teca en diferentes sustratos orgánicos
}

Recebido: 03/04/2021 | Revisado: 11/04/2021 | Aceito: 12/04/2021 | Publicado: 25/04/2021

\author{
Lucas Gabriel Guse \\ ORCID: https://orcid.org/0000-0001-7075-3751 \\ Universidade Federal do Maranhão, Brasil \\ E-mail: lucaasguse@ hotmail.com \\ Ana Carolinne Dias Leão \\ ORCID: https://orcid.org/0000-0002-1654-0090 \\ Universidade Federal do Maranhão, Brasil \\ E-mail: carolinnefla_diasleao@hotmail.com \\ Luisa Julieth Parra-Serrano \\ ORCID: https://orcid.org/0000-0002-9796-1988 \\ Universidade Federal do Maranhão, Brasil \\ E-mail: luisa.jps@ufma.br \\ Mariléia Barros Furtado \\ ORCID: https://orcid.org/0000-0003-4696-2136 \\ Universidade Federal do Maranhão, Brasil \\ E-mail: marileia.furtado@ufma.br \\ Maryzélia Furtado de Farias \\ ORCID: https://orcid.org/0000-0001-9078-1354 \\ Universidade Federal do Maranhão, Brasil \\ E-mail: maryzelia.furtado@ufma.br
}

\begin{abstract}
Resumo
A teca (Tectona grandis L.f.) é uma árvore de origem asiática que vem sendo extensamente implantada no Brasil, porém, seu uso é restrito devido à dificuldade na produção de mudas, sendo a utilização de compostos orgânicos uma alternativa para sua produção. Portanto, objetivou-se avaliar o uso de esterco de caprino, caule decomposto de babaçu e bokashi como componentes de substratos na produção de mudas de teca. $\mathrm{O}$ experimento foi conduzido em casa de vegetação com duração de 60 dias. Foi utilizado delineamento inteiramente casualizado, com dez tratamentos, compostos por esterco caprino (EC1, EC2 e EC3), caule descomposto de babaçu (CB1, CB2 e CB3) e bokashi (BO1, BO2 e BO3), com proporções de 45, 30 e $15 \%$, respectivamente, associados ao solo, contendo um tratamento controle com 100\% solo (Latossolo Vermelho Amarelo distrófico), e quatro repetições. Foram avaliadas a altura da parte aérea, o diâmetro de colo, o número de folhas e o Índice de Qualidade de Dickson. Os dados foram analisados através do software InfoStat $\odot$ e as médias comparadas pelo teste Duncan. O uso de bokashi e esterco de caprino como componentes de substratos na produção de mudas de teca possibilita desempenhos favoráveis. A utilização de no mínimo 15\% de bokashi ou de esterco caprino permitiram a formação de mudas de melhor qualidade.
\end{abstract}

Palavras-chave: Babaçu; Bokashi; Esterco caprino; Tectona grandis.

\begin{abstract}
Teak (Tectona grandis L.f.) is a tree of Asian origin that has been widely planted in Brazil; however, its use is restricted due to the difficulty in producing seedlings, being the use of organic compounds an alternative for its production. Therefore, the objective was to evaluate the use of goat manure, decomposed trunk of babassu palm and bokashi as components of substrates in the production of teak seedlings. The experiment was carried out in a nursery for 60 days. A completely randomized design was used, with ten treatments, substrates composed of goat manure (EC1, EC2 and EC3), decomposed trunk of babassu palm (CB1, CB2 and CB3) and bokashi (BO1, BO2 and BO3), with proportions of 45,30 and $15 \%$, respectively, associated with the soil, with a control treatment with $100 \%$ soil (Latosol Red-Yellow Dystrophic), and four repetitions. The height of the aerial part, the diameter of the stem at the neck level, the number of leaves and the Dickson Quality Index were evaluated. The data were analyzed using the InfoStat (C) software and the averages compared using the Duncan test. The use of bokashi and goat manure as substrate components for the production of teak seedlings allows favorable yields. The use of at least $15 \%$, bokashi or goat manure allows the formation of better quality seedlings.
\end{abstract}

Keywords: Babassu; Bokashi; Goat manure; Tectona grandis.

\section{Resumen}

La teca (Tectona grandis L.f.) es un árbol de origen asiático que ha sido ampliamente plantado en Brasil, sin embargo, su uso es restringido debido a la dificultad para producir plántulas, siendo la utilización de compuestos orgánicos una 
alternativa para su producción. Por lo tanto, el objetivo fue evaluar el uso de estiércol caprino, tronco descompuesto de palmera babasú y bocashi como componentes de sustratos en la producción de plántulas de teca. El experimento se realizó en un vivero durante 60 días. Se utilizó un diseño completamente al azar, con diez tratamientos, sustratos compuestos por estiércol caprino (EC1, EC2 y EC3), tronco descompuesto de palmera babasú (CB1, CB2 y CB3) y bocashi (BO1, BO2 y BO3), con proporciones de 45, 30 y 15\%, respectivamente, asociados al suelo, con un tratamiento control con $100 \%$ de suelo (Latosol Rojo-Amarillo Distrófico), y cuatro repeticiones. Se evaluó la altura de la parte aérea, el diámetro del tallo a nivel del cuello, el número de hojas y el Índice de Calidad de Dickson. Los datos se analizaron mediante el software InfoStat (C) y las medias se compararon mediante la prueba de Duncan. El uso de bocashi y de estiércol de caprino como componentes de sustratos para la producción de plántulas de teca permite rendimientos favorables. El uso de mínimo $15 \%$, bocashi o estiércol caprino permite la formación de plántulas de mejor calidad.

Palabras clave: Babasú; Bocashi; Estiércol caprino; Tectona grandis.

\section{Introdução}

A teca (Tectona grandis L.f.) é uma árvore originária do continente asiático e seu cultivo vem expandindo no Brasil, principalmente nas regiões Centro-Oeste e Norte, sendo considerada insubstituível, principalmente, para a indústria naval e produção de móveis finos, devido à nobreza de sua madeira (Rocha et al., 2015), que carrega excelentes propriedades físicas, mecânicas e estéticas (Figueiredo, 2001; Blanco-Flórez, et al. 2014).

Apesar da teca ser uma ótima alternativa para um desenvolvimento sustentável através da redução da pressão sobre florestas nativas, sua propagação é feita por sementes e sua germinação é lenta e irregular devido à elevada dormência do fruto, também chamado de diásporo, fazendo com que esta seja uma das principais limitações do seu uso em grandes plantios de produtores de madeira (Ferreira, et al., 2016).

Segundo Dabral (1967), apud Dias et al. (2009) o fruto é do tipo drupa, tetralocular, podendo conter até quatro sementes, e estas são protegidas por uma grossa camada de endocarpo e mesocarpo impermeáveis, sendo necessário o rompimento dessa barreira física para a germinação das sementes, através da quebra de dormência.

Deste modo, a prioridade, após a germinação das sementes, gira em torno da qualidade das mudas, que reflete diretamente na produtividade e na qualidade do produto final. Uma alternativa para a produção de mudas é a utilização de resíduos orgânicos na composição dos substratos (Cruz-Crespo et al. 2013), pois além de fornecerem nutrientes e matéria orgânica, melhoram o arejamento, a capacidade de retenção de água e estimulam o desenvolvimento de microrganismos benéficos no solo (Wendling 2002; Caldeira et al., 2008; Gomes \& De Paiva, 2013). Dentre os compostos orgânicos ainda não estudados na produção de mudas florestais, destacam-se o esterco caprino, o caule decomposto de babaçu e o bokashi.

O esterco caprino não é comumente utilizado como componente de substratos para produção de mudas florestais, porém, devido à sua alta disponibilidade na região, seu baixo custo de aquisição e seus atributos químicos, há uma necessidade de novos estudos que incentivem sua utilização. Diversos autores relatam benefícios no desenvolvimento de mudas agrícolas pela incorporação do esterco caprino, o qual promove a melhoria das qualidades físicas, químicas e biológicas dos substratos (Araújo et al., 2010, Notaro et al., 2012, Amorim et al., 2018).

O babaçu (Attalea speciosa sin. Orbignya phalerata) é uma palmeira considerada característica da formação vegetal chamada Mata dos Cocais, e vem sendo alvo de pesquisas avançadas para a produção de bioenergia por combustão direta e produção de carvão vegetal (De Paula Protásio et al. 2014), porém, há poucos estudos que mostram o potencial do seu caule decomposto na produção vegetal.

O bokashi é um adubo orgânico concentrado enriquecido com microrganismos eficazes (Magrini et al., 2011, Lima et al., 2015) empregado como fonte de matéria orgânica e nutrientes (Quiroz \& Céspedes, 2019), e seu uso é considerado uma das formas mais antigas de reciclagem de resíduos orgânicos, gerando bons resultados, porém, informações a respeito do seu uso na produção de mudas florestais são escassas.

Diante do exposto e a carência de trabalhos com produção de mudas florestais utilizando os componentes de 
substratos mencionados, o objetivo deste trabalho foi avaliar o uso de esterco de caprino, caule decomposto de babaçu e bokashi como componentes de substratos na produção de mudas de teca.

\section{Metodologia}

\section{Condições experimentais}

Este estudo trata-se de uma pesquisa quantitativa e experimental (Gerhardt \& Silveira, 2009), realizada em casa de vegetação, sob telado com 50\% de sombreamento, no período de maio a agosto de 2019 no Município de Chapadinha-MA, situado a $03^{\circ} 44^{\prime} 30^{\prime \prime}$ Sul de latitude e $43^{\circ} 21^{\prime} 33^{\prime \prime}$ Oeste de longitude, com altitude de $105 \mathrm{~m}$, de acordo com o software SPRING® 4.3.3 (INPE 2010). A classificação climática de acordo com Köppen e Geiger é Aw, considerado zona tropical com inverno seco e verão chuvoso (Alvares et al., 2013), com uma estação chuvosa que se estende de janeiro a junho e uma estação seca com déficit hídrico de julho a dezembro e precipitação anual média de $2100 \mathrm{~mm}$ (De Moura-Silva et al., 2016).

O delineamento utilizado foi inteiramente casualizado (DIC), com dez tratamentos e quatro repetições, com quatro plantas por repetição, totalizando 160 mudas de teca. Para a composição dos diferentes substratos (tratamentos), foi utilizado esterco caprino, caule decomposto de babaçu e bokashi, em diferentes proporções, associados ao solo caracterizado como um Latossolo Vermelho Amarelo distrófico, obtido na profundidade de $0-20 \mathrm{~cm}$ (Tabela 1), e que apresentam as seguintes características químicas: $\mathrm{pH}=5,1, \mathrm{P}=11,5 \mathrm{mg} \mathrm{dm}^{-3}, \mathrm{~K}=33 \mathrm{mg} \mathrm{dm}^{-3} \mathrm{e} \mathrm{N}=1,35 \mathrm{~g} \mathrm{~kg}^{-1}$; o esterco caprino possui $\mathrm{pH}=7,8, \mathrm{P}$ $=2,6 \mathrm{~g} \mathrm{~kg}^{-1}, \mathrm{~K}=16,8 \mathrm{~g} \mathrm{~kg}^{-1} \mathrm{e} \mathrm{N}=12,8 \mathrm{~g} \mathrm{~kg}^{-1}$ (Da Silva Sá et al. 2014, Souza et al. 2017); o caule decomposto de babaçu possui $\mathrm{pH}=5,3, \mathrm{P}=33 \mathrm{mg} \mathrm{kg}^{-1}, \mathrm{~K}=3,63$ cmolc kg-1 e N = 5,88 $\mathrm{g} \mathrm{kg}^{-1}$ (Oliveira et al. 2018); e o bokashi possui $\mathrm{pH}=8, \mathrm{P}=$ $31,1 \mathrm{~g} \mathrm{~kg}^{-1}, \mathrm{~K}=16,3 \mathrm{~g} \mathrm{~kg}^{-1} \mathrm{e} \mathrm{N}=29 \mathrm{~g} \mathrm{~kg}^{-1}$.

Tabela 1. Composição dos substratos e identificação dos tratamentos para a produção de mudas de teca empregando esterco caprino (EC), caule decomposto de babaçu (CB) e bokashi (BO) associados ao solo (SO).

\begin{tabular}{ccccc}
\hline Tratamentos & Solo (\%) & $\begin{array}{c}\text { Esterco caprino } \\
(\%)\end{array}$ & $\begin{array}{c}\text { Caule decomposto de } \\
\text { babaçu (\%) }\end{array}$ & Bokashi (\%) \\
\hline EC1 & 55 & 45 & - & - \\
EC2 & 70 & 30 & - & - \\
EC3 & 85 & 15 & - & - \\
CB1 & 55 & - & 45 & - \\
CB2 & 70 & - & 30 & - \\
CB3 & 85 & - & 15 & - \\
BO1 & 55 & - & - & 45 \\
BO2 & 70 & - & - & 30 \\
BO3 & 85 & - & - & - \\
SO & 100 & - & - & 15 \\
\hline
\end{tabular}

Fonte: Autores.

\section{Preparo dos componentes dos substratos}

Os componentes dos substratos precisaram de preparos para sua utilização. O esterco caprino passou por estabilização biológica no processo de curtimento durante 60 dias; o material decomposto da palmeira de babaçu foi adquirido na região e homogeneizado em peneira de malha de $3 \mathrm{~mm}$ para facilitar a mistura com o solo; e o bokashi foi produzido no local do experimento seguindo os procedimentos sugeridos por Siqueira e Siqueira (2013) que consistem na captura e ativação dos 
microrganismos eficazes, seleção e quantificação dos ingredientes e preparo final do componente.

Para a captura dos microrganismos eficazes foram cozidos $700 \mathrm{~g}$ de arroz sem sal e sem óleo; este foi colocado em uma bandeja de madeira que foi coberta com uma tela fina para proteção; a bandeja foi colocada no solo, sob a mata, próximo a sua borda; a tela que protege o arroz foi coberta com serapilheira (matéria orgânica) da área. Entre 5 e 8 dias depois, quando o arroz continha bolores de diversas cores, foram retiradas para o uso as partes mofadas de coloração azulada, amarelada, rosada e alaranjada, descartando coloração cinza, marrom e preta, pois estas colorações poderiam comprometer o processo de fermentação e posteriormente a qualidade do composto.

As partes mofadas na coloração de interesse foram colocadas em um balde com dez litros de água misturada com $1 \mathrm{~kg}$ de melado de cana-de-açúcar; a mistura foi, então, colocada em um recipiente com tampa, sendo aberto a cada dois dias para que o gás produzido fosse liberado; entre 10 e 15 dias depois, quando não havia mais produção de gás, os microrganismos estavam ativados e prontos para serem utilizados.

Para produzir $100 \mathrm{~kg}$ de bokashi, foram utilizados $30 \mathrm{~kg}$ de farelo de arroz, $30 \mathrm{~kg}$ de farelo de trigo, $3 \mathrm{~kg}$ de farinha de carne e ossos, $35 \mathrm{~kg}$ de torta de babaçu e $2 \mathrm{~kg}$ de calcário para constituir o material seco; além disso, foram utilizados 1 litro de microrganismos eficazes, 1 litro de caldo de cana e 30 litros de água para constituir a solução líquida.

Os ingredientes foram, então, misturados, dispostos em formato de canteiro com $20 \mathrm{~cm}$ de altura; a mistura foi coberta com plástico durante os dois primeiros dias para que se iniciasse o processo de fermentação; a partir do segundo dia a mistura foi revirada diariamente até o sétimo dia, sendo que a partir do quarto dia a altura foi reduzida para $10 \mathrm{~cm}$.

No sétimo dia, o bokashi estava totalmente seco, e, para sua utilização como componente dos substratos, passou por um processo de estabilização biológica, onde foi adicionado palha de arroz ao bokashi, na proporção de 30\%, para melhorar a aeração e dispor a mistura em canteiro coberto com $10 \mathrm{~cm}$ de altura, onde foi molhado diariamente até estabilizar sua temperatura com o ambiente.

\section{Produção e avaliação das mudas}

As mudas de teca (Tectona grandis) foram produzidas a partir de sementes, cujos frutos da espécie foram coletados no segundo semestre de 2018 em uma propriedade local. Para o processo de quebra de dormência dos frutos, foram seguidas as recomendações de Figueiredo et al. (2005), colocando os diásporos imersos em água durante três noites e expondo-os ao sol durante três dias; posteriormente, realizou-se a semeadura em um canteiro com areia lavada.

Após as mudas atingirem $5 \mathrm{~cm}$ de altura, estas foram transferidas para sacos plásticos próprios para mudas florestais, de tamanho $15 \times 22 \mathrm{~cm}, 0,20$ micras de espessura e capacidade volumétrica de 1,4 L. Os sacos foram, então, preenchidos com seus respectivos substratos e dispostos em bancadas a $50 \mathrm{~cm}$ do solo, onde permaneceram durante 60 dias em casa de vegetação, sendo regadas diariamente.

As mudas foram avaliadas a cada 15 dias, durante dois meses, e no final do experimento, aos 60 dias. As características avaliadas nas mudas foram: diâmetro de colo (D), altura (H), número de folhas (NF) e índice de qualidade de Dickson (IQD).

A altura da parte aérea da muda foi medida com o uso de uma régua graduada em milímetros, a partir do nível do substrato até a inserção da última folha; para diâmetro de colo foi utilizado um paquímetro digital, medido na altura do colo da planta; e o IQD foi calculado através da equação 1:

$$
I Q D=\frac{M S T}{\frac{H}{D}+\frac{M S P A}{M S R}}
$$


Em que, MST é a matéria seca total $(\mathrm{g})$; H é a altura $(\mathrm{cm})$; D é o diâmetro $(\mathrm{mm})$; MSPA é a matéria seca da parte aérea (g); e MSR é a matéria seca da raiz (g). Para a obtenção da MSPA e MSR, foi feita a secagem, em estufa a $70^{\circ} \mathrm{C}$ durante 72 horas, da parte aérea e da raiz das mudas, respectivamente, e com a soma destes foi obtida a MST. A análise de variância dos dados foi realizada através do software estatístico InfoStat@ (2018) e as médias comparadas pelo teste Duncan a 5\% de significância.

\section{Resultados e Discussão}

Os substratos apresentaram efeito significativo $(\mathrm{P}<0,05)$ para todas as variáveis analisadas (Tabela 2).

Tabela 2. Resumo da análise de variância individual (valor de F) de diâmetro (D), altura (H), número de folhas (NF) e índice de qualidade de Dickson (IQD) de mudas de teca (Tectona grandis) com diferentes substratos.

\begin{tabular}{cccccc}
\hline Fontes de Variação & Graus de Liberdade & $\mathrm{D}(\mathrm{mm})$ & $\mathrm{H}(\mathrm{cm})$ & $\mathrm{NF}$ & $\mathrm{IQD}$ \\
\hline Tratamentos & 9 & $20,460^{*}$ & $6,897^{*}$ & $4,08^{*}$ & $30,600^{*}$ \\
Resíduo & 30 & 2,13 & 2,13 & 2,13 & 2,13 \\
Total & 39 & & & & \\
\hline CV $(\%)$ & - & 17,97 & 20,95 & 20,72 & 28,57 \\
\hline
\end{tabular}

*Significativo a $95 \%$ de probabilidade pelo teste F. Fonte: Autores.

A fim de homogeneizar ainda mais as análises, visto que as mudas não iniciaram o experimento com mesmas alturas e diâmetros, foi calculado apenas o incremento destas variáveis, indicando somente o desenvolvimento das mesmas. Pode-se observar, na Tabela 3, que o tratamento controle obteve os menores médias de crescimento em diâmetro, altura e índice de qualidade de Dickson, deixando clara a inviabilidade deste para produção de mudas da espécie, necessitando a adição de fontes ricas em nutrientes.

Tabela 3. Médias de crescimento em diâmetro (D) e em altura (H), número de folhas (NF) e índice de qualidade de Dickson (IQD) de mudas de teca (Tectona grandis) com diferentes substratos.

\begin{tabular}{ccccc}
\hline \multirow{2}{*}{ Tratamento $^{1}$} & \multicolumn{3}{c}{ Variável } \\
\cline { 2 - 5 } & $\mathrm{D}(\mathrm{mm})$ & $\mathrm{H}(\mathrm{cm})$ & $\mathrm{NF}$ & IQD \\
\hline EC1 & $3,835^{\mathrm{ab}}$ & $19,343^{\mathrm{ab}}$ & $7,425^{\mathrm{ab}}$ & $0,790^{\mathrm{b}}$ \\
$\mathrm{EC} 2$ & $4,104^{\mathrm{ab}}$ & $20,781^{\mathrm{a}}$ & $8,625^{\mathrm{ab}}$ & $1,106^{\mathrm{a}}$ \\
$\mathrm{EC} 3$ & $4,431^{\mathrm{a}}$ & $19,125^{\mathrm{ab}}$ & $6,625^{\mathrm{b}}$ & $1,021^{\mathrm{ab}}$ \\
$\mathrm{CB} 1$ & $3,096^{\mathrm{b}}$ & $14,750^{\mathrm{ab}}$ & $9,875^{\mathrm{ab}}$ & $0,509^{\mathrm{c}}$ \\
CB2 & $2,985^{\mathrm{b}}$ & $12,969^{\mathrm{b}}$ & $10,875^{\mathrm{a}}$ & $0,504^{\mathrm{c}}$ \\
CB3 & $3,387^{\mathrm{b}}$ & $15,500^{\mathrm{ab}}$ & $9,250^{\mathrm{ab}}$ & $0,794^{\mathrm{b}}$ \\
BO1 & $4,423^{\mathrm{a}}$ & $20,469^{\mathrm{a}}$ & $6,875^{\mathrm{b}}$ & $0,923^{\mathrm{ab}}$ \\
BO2 & $4,394^{\mathrm{a}}$ & $21,157^{\mathrm{a}}$ & $8,000^{\mathrm{ab}}$ & $1,074^{\mathrm{a}}$ \\
BO3 & $4,485^{\mathrm{a}}$ & $19,406^{\mathrm{ab}}$ & $8,250^{\mathrm{ab}}$ & $1,128^{\mathrm{a}}$ \\
SO & $2,008^{\mathrm{c}}$ & $10,969^{\mathrm{b}}$ & $7,750^{\mathrm{ab}}$ & $0,303^{\mathrm{c}}$ \\
\hline
\end{tabular}

Médias seguidas de letras iguais, nas colunas, não diferem estatisticamente entre si pelo teste Duncan $(\mathrm{P}<0,05) .{ }^{1} \mathrm{EC} 1=45 \%$ de esterco caprino; $\mathrm{EC} 2=30 \%$ de esterco caprino; $\mathrm{EC} 3=15 \%$ de esterco caprino; $\mathrm{CB} 1=45 \%$ de caule decomposto de babaçu; $\mathrm{CB} 2=30 \%$ de caule decomposto de babaçu; $\mathrm{CB} 3=15 \%$ de caule decomposto de babaçu; $\mathrm{BO} 1=45 \%$ de bokashi; $\mathrm{BO} 2=30 \%$ de bokashi; $\mathrm{BO} 3=15 \%$ de bokashi; $\mathrm{SO}=100 \%$ de solo. Fonte: Autores. 
Os tratamentos compostos por bokashi em diferentes proporções apresentaram os melhores resultados na maioria das variáveis analisadas, evidenciando o potencial deste substrato para produção de mudas. Este pode ser explicado pelo incremento dos microrganismos benéficos que este material proporciona, criando condições favoráveis para o desenvolvimento da microbiota presente no solo, como bactérias fixadoras de nitrogênio e fungos, que, correlacionado com suas quantidades balanceadas de macro e micronutrientes disponíveis, propicia um ambiente equilibrado do ponto de vista físico, químico e biológico para o desenvolvimento vegetal (Siqueira \& Siqueira 2013). Estes autores recomendam a aplicação de baixas dosagens de bokashi na produção vegetal, exemplificadas nos resultados superiores nos tratamentos que continham menor proporção de bokashi (15 e 30\%) devido a este ser um composto concentrado e eficaz.

Foi observado no período experimental, que, embora o número de folhas das mudas produzidas nos substratos compostos por bokashi tenha sido inferior, estas eram maiores, gerando novas hipóteses para a realização de novos trabalhos que visem correlacionar o número de folhas das mudas de teca com sua área foliar.

Santos et al. (2019) verificaram que doses crescentes de bokashi favorecem as características morfométricas foliares, aumentando a área foliar das mudas de guabiroba do campo (Campomanesia adamantium), mas acima de $15 \mathrm{~g}$ de bokashi foi prejudicial para a sobrevivência das mudas. O incremento na área foliar proporcionado pelo uso do bokashi está associado ao seu alto teor de nutrientes, como nitrogênio, fosforo e potássio.

O diâmetro do colo é um item essencial para a avaliação de mudas florestais, pois, quando dentro da mesma espécie, plantas de maior diâmetro apresentam maior porcentagem de sobrevivência, visto que estas variáveis possuem forte correlação entre si (Carneiro 1995, Souza et al., 2006). O diâmetro das mudas variou de 1,7 a 5,2 $\mathrm{mm}$ e os substratos que proporcionaram maior crescimento nesta variável foram BO3, EC3, BO1 e BO2.

O diâmetro das mudas assemelhou-se ao daquelas obtidas por Trazzi et al. (2013) com mudas de 90 dias após repicagem, sendo 5,8 $\mathrm{mm}$ neste e 4,5 $\mathrm{mm}$ no presente estudo. Possivelmente, com maior quantidade de dias, as mudas continuariam tendo bom desempenho, podendo alcançar diâmetros ainda superiores.

A altura das plantas apresentou valores entre 9,6 e $27 \mathrm{~cm}$, sendo as maiores médias alcançadas pelos substratos BO2, EC2 e BO1. O crescimento das mudas produzidas com $30 \%$ de caule decomposto de babaçu foi semelhante às mudas desenvolvidas no tratamento controle.

As maiores médias de altura do experimento foram entre 20,5 e 21,2 cm, estas se mostraram bem desenvolvidas quando comparadas a outros trabalhos realizados com a mesma espécie florestal, visto que as mudas da presente pesquisa foram avaliadas apenas até 60 dias após repicagem, e os demais trabalhos avaliaram 90 e 120 dias após, obtendo maiores médias em altura de 11,7 cm (Caldeira et al., 2012), 19,9 cm (Gomes et al., 2013), 22,3 (Trazzi et al., 2013) e entre 34,7 e 35,9 $\mathrm{cm}$ (Trazzi et al., 2014), mostrando que os substratos do presente estudo disponibilizaram melhores condições de crescimento para as mudas em relação ao tempo de condução do experimento.

Foi observado, durante todo o período experimental, que as mudas de teca mantinham constante o número de folhas, com quedas e renovações, independentes do substrato em que estavam inseridas. No fim do experimento, o número de folhas nas mudas variou de 6,6 a 10,9, estando a maior quantidade de folhas presente nos tratamentos compostos por caule decomposto de babaçu.

Fernandez (2002) e Costa et al. (2005) em trabalhos realizados com mudas de Mangaba (Hancornia speciosa) e Genipapo (Genipa americana) respectivamente, observaram que a utilização de esterco bovino na produção destas, influenciava o número de folhas presente nas plantas. Pinheiro et al. (2018) e Cavalcante et al. (2016) trabalhando com produção de mudas de sabiá (Mimosa caesalpiniaefoli) e gliricídia (Gliricidia sepium) respetivamente observaram que os substratos formulados com estercos de animais promoveram a formação de mudas com maior número de folhas, esse efeito positivo é associado ao incremento da matéria orgânica proporcionado pelos compostos. 
Os valores de IQD calculados variaram de 0,20 a 1,28, gerando um coeficiente de variação de $28,6 \%$, sendo as maiores médias observadas nos tratamentos $\mathrm{BO}$, EC2 e BO2, e as menores nos tratamentos $\mathrm{CB} 1, \mathrm{CB} 2$ e SO.

Embora as médias de diâmetro, altura, número de folhas e IQD obtidas nos tratamentos com caule decomposto de babaçu tenham sido inferiores, suas mudas mostraram-se mais homogêneas. Isto pode ser explicado devido ao fato de que, durante o experimento, o substrato mantinha-se mais úmido durante todo o dia, devido ao tamanho das suas partículas e sua baixa densidade, visto que o material foi peneirado com malha de $3 \mathrm{~mm}$, obtendo partículas grandes que proporcionaram um efeito de "esponja".

Portanto, a homogeneidade na produção das mudas foi alcançada devido às melhorias nas qualidades físicas do substrato com caule decomposto de babaçu, porém, seu desempenho não foi superior em função da baixa quantidade de nutrientes presentes no material, sendo recomendada uma fonte extra de nutrientes para complementar o uso deste componente.

Caldeira et al. (2012) avaliando o desenvolvimento de mudas de teca de 120 dias após repicagem, observaram que $40 \%$ de biossólido proporcionou mudas com altura média de 11,7, superior às demais, este tratamento, juntamente com $80 \%$ de biossólido gerou médias entre 9,9 e $10 \mathrm{~mm}$ de diâmetro e este último tratamento, de $80 \%$ de biossólido, concedeu mudas com maiores índices de qualidade de Dickson, com média de 2,23.

Gomes et al. (2013) verificaram o efeito da adição de lodo de esgoto na produção de mudas de teca de 120 dias após repicagem, e puderam observar que o substrato composto por $60 \%$ de lodo de esgoto proporcionou mudas com 19,87 cm de altura, o substrato com $40 \%$ de lodo gerou maior diâmetro, de $9,41 \mathrm{~mm}$, e todos os tratamentos, exceto o tratamento contendo apenas $20 \%$ de lodo de esgoto, ocasionaram IQD semelhantes, variando de 1,68 a 1,97.

Avaliando o uso de esterco bovino, cama de frango e esterco de codorna em diferentes proporções na produção de mudas de teca com 90 dias após repicagem, Trazzi et al. (2013) observaram que 35\% de cama de frango apresentou maiores médias tanto em altura quanto em diâmetro de colo, obtendo valores de $22,31 \mathrm{~cm}$ e 5,79 mm, respectivamente, e os valores de IQD variaram de 0,40 a 1,04, sendo as maiores médias encontradas nos tratamentos de 35 e $25 \%$ de cama de frango e $25 \%$ de esterco de codorna.

Trazzi et al. (2014), utilizando diferentes compostos renováveis na produção de mudas de teca com até 120 dias após repicagem, verificaram que os tratamentos em que o biossólido estava associado à casca de arroz carbonizada proporcionaram maior crescimento em altura, obtendo médias de 34,7 a $35,9 \mathrm{~cm}$, as maiores médias em diâmetro foram obtidas nos tratamentos em que o biossólido estava associado à 20 e $40 \%$ de fibra de coco triturada, atingindo diâmetros de 7,4 mm, e os maiores índices de qualidade de Dickson foram alcançados nas mudas produzidas com biossólido associado à 40 e $60 \%$ de fibra de coco, atingindo valores de 2,34.

De um modo geral, o índice de qualidade de Dickson das mudas foi superior ao encontrado por Trazzi et al. (2013), mesmo estas sendo avaliadas com 30 dias a menos, indicando que os substratos compostos por esterco caprino, caule decomposto de babaçu e bokashi, proporcionam mudas de melhor qualidade quando comparadas a mudas produzidas com esterco bovino, esterco de codorna e cama de frango nas proporções utilizadas pelos autores.

Assim, evidencia-se a importância da utilização da matéria orgânica nos substratos, visto que mantem as características adequadas para o desenvolvimento das mudas, como manutenção da umidade, porosidade e disponibilidade de nutrientes.

\section{Conclusão}

O uso de no mínimo $15 \%$ de bokashi ou de esterco caprino possibilita a produção de mudas de teca de boa qualidade. O substrato com caule decomposto de babaçu promove redução na qualidade das mudas de teca, não sendo, portanto, 
recomendável sua utilização sem a adição de uma fonte extra de nutrientes. O Latossolo Vermelho Amarelo distrófico sem incorporação de resíduos orgânicos não fornece as condições apropriadas para a produção de mudas de teca. É recomendável o desenvolvimento de novos estudos que avaliem os substratos analisados neste experimento associados a outras fontes orgânicas, em diferentes proporções, visando identificar quantidades mais específicas que proporcionem mudas de melhor qualidade e com melhores características de desenvolvimento inicial.

\section{Referências}

Alvares, C. A., Stape, J. L., Sentelhas, P. C., Gonçalves, J. D. M., \& Sparovek, G. (2013). Köppen's climate classification map for Brazil. Meteorologische Zeitschrift, 22(6), 711-728. https://doi.org/10.1127/0941-948/2013/0507

Amorim, D. J., dos Santos, J. C. C., Parra-Serrano, L. J., de Farias, M. F., \& Furtado, M. B. (2018). Multidimensional analysis associated with growth analysis in the selection of organic substrates for the production of tomato seedlings. Journal of Agricultural Science, $10(11)$, 326-336. https://doi.org/10.5539/jas.v10n11p326

Araújo, W. B. M., Alencar, R. D., Mendonça, V., de Medeiros, E. V., de Carvalho Andrade, R., \& de Araújo, R. R. (2010). Esterco caprino na composição de substratos para formação de mudas de mamoeiro. Ciência e Agrotecnologia, 34(1), 68-73.https://doi.org/10.1590/S1413-70542010000100008

Blanco-Flórez, J. B., Trugilho, P. F., Lima, J. T., Hein, P. R. G., \& Silva, J. R. M. D. (2014). Caracterización de la madera joven de Tectona grandis L. f. plantada en Brasil. Madera y bosques, 20(1), 11-20.

Caldeira, M. V. W., FENILLI, T. A. B., \& HARBS, R. M. P. (2008). Composto orgânico na produção de mudas de aroeira-vermelha. Scientia Agraria, 9(1), 027-033.

Caldeira, M. V. W., Delarmelina, W. M., Lübe, S. G., Gomes, D. R., de Oliveira Gonçalves, E., \& Alves, A. F. (2012). Biossólido na composição de substrato para a produção de mudas de Tectona grandis. Floresta, 42(1), 77-84. http://dx.doi.org/10.5380/rf.v42i1.26302

Carneiro, J. G. D. A. (1995). Produção e controle de qualidade de mudas florestais. UFPR / FUPEF; UENF. 451p.

Cavalcante, A. C. P., Cavalcante, A. G., da Silva, M. J. R., \& da Costa Araújo, R. (2016). Produção de mudas de gliricídia com diferentes substratos orgânicos. Agrarian, 9(33), 233-240.

Costa, M. C., de Figueiredo, M. C., Albrecht, J. M. F., \& Coelho, M. D. F. B. (2005). Substratos para produção de mudas de jenipapo (Genipa americana L.). Pesquisa Agropecuária Tropical, 35(1), 19-24.

Cruz-Crespo, E., Can-Chulim, A., Sandoval-Villa, M., Bugarin-Montoya, R., Robles-Bermudez, A., \& Juarez-Lopez, P. (2013). Sustratos en la horticultura. Revista Bio Ciencias. 2(2), 17-26.

Dabral, S. L. (1967) Extraction of teak seeds from fruits, their storage and germination. Indian Forester, Dhera Dun. 102 (10), $650-658$.

Da Silva Sá, F. V., Bertino, A. M. P., Ferreira, N. M., Bertino, A. M. P., da Silva Soares, L., \& de Mesquita, E. F. (2017). Formação de mudas de maracujazeiro amarelo com diferentes doses de esterco caprino e volumes do substrato. Magistra, 26(4), $482-492$.

De Moura-Silva, A. G., Aguiar, A. D. C. F., de Moura, E. G., \& Jorge, N. (2016). Influence of soil cover and N and K fertilization on the quality of biofortified QPM in the humid tropics. Journal of the Science of Food and Agriculture, 96(11), 3807-3812. https://doi.org/10.1002/jsfa.7574

De Paula Protásio, T., Trugilho, P. F., da Silva César, A. A., Napoli, A., de Melo, I. C. N. A., \& da Silva, M. G. (2014). Babassu nut residues: potential for bioenergy use in the North and Northeast of Brazil. SpringerPlus, 3(1), 1-14. https://doi.org/10.1186/2193-1801-3-124

Dias, J. R. M., Caproni, A. L., Wadt, P. G. S., Silva, L. M. D., Tavella, L. B., \& Oliveira, J. P. D. (2009). Quebra de dormência em diásporos de teca (Tectona grandis Lf). Acta Amazonica, 39(3), 549-554. http://dx.doi.org/10.1590/S0044-59672009000300009

Ferreira, R. Q. D. S., Camargo, M. O., Junior, M. R. S., de Souza, P. B., \& de Oliveira, L. M. (2016). Choque térmico na superação da dormência de diásporos de Tectona grandis L. f. Scientia Plena, 12(3). http://dx.doi.org/10.14808/sci.plena.2016.030201

Figueiredo, E. O. (2001). Reflorestamento com teca (Tectona grandis L. f.) no estado do Acre. Embrapa Acre-Documentos (INFOTECA-E).

Figueiredo, E. O., Oliveira, L. D., \& Barbosa, L. K. F. (2005). Teca (Tectona grandis L. f.): principais perguntas do futuro empreendedor florestal. Rio Branco: Embrapa Acre.

Gerhardt, T. E. \& Silveira, D. T. (2009). Métodos de pesquisa. Editora da UFRGS, Brasil.

Gomes, D. R., Caldeira, M. V. W., Delarmelina, W. M., Gonçalves, E. D. O., \& Trazzi, P. A. (2013). Lodo de esgoto como substrato para produção de mudas de Tectona grandis L. Cerne, 19(1), 123-131.

Paiva, H. N., \& Gomes, J. M. (2013). Viveiros florestais: propagação sexuada. Editora UFV, Brasil.

Instituto Nacional De Pesquisas Espaciais (INPE). (2010). Manual do software SPRING (Sistema de processamento de informações georreferenciadas). v. 4.3.3. 
Lima, C. E. P., Fontenelle, M. R., Silva, L. R. B., Soares, D. C., Moita, A. W., Zandonadi, D. B., \& Lopes, C. A. (2015). Short-term changes in fertility attributes and soil organic matter caused by the addition of EM Bokashis in two tropical soils. International Journal of Agronomy. https://doi.org/10.1155/2015/754298

Macedo, V. R. A., Guiscem, J. M., Chaves, A. M. S., Monteiro, A. L. R., Bitu, P. I. M., \& Pinheiro, G. V. (2011). 11710-Avaliação do húmus do caule de Palmeira do Babaçu como substrato. I Característica química e sua viabilidade na produção de mudas de alface. Cadernos de Agroecologia, 6(2).

Magrini, F. E., Sartori, V. C., Finkler, R., Torves, J., \& Venturin, L. (2011). Características químicas e avaliação microbiológica de diferentes fases de maturação do biofertilizante Bokashi. Agrarian, 4(12), 146-151.

Notaro, K. A., Souza, B. M., Silva, A. O., da Silva, M. M., Medeiros, E. V., \& Duda, G. P. (2012). População microbiana rizosférica, disponibilidade de nutrientes e crescimento de pinheira, em substratos com resíduos orgânicos. Revista Brasileira de Ciências Agrárias, 7, 770-776. https://10.5039/agraria.v7isa201

Oliveira, A. R. F., Moura, M. S., Cordeiro, K. V., Machado, N. A. F., \& Silva-Matos, R. R. S. (2017). Caracterização química de substratos formulados a partir de caule decomposto de babaçu. In Trabalho apresentado no Congresso Técnico Científico da Engenharia e da Agronomia CONTECC.

Pinheiro, J. I., de Sousa Oliveira, L., de Sousa, A. M., Garcia, K. G. V., \& Lima, L. A. (2018). Mudas de Mimosa caesalpiniaefolia Benth (Leguminosae: Mimosoideae) cultivadas em substratos orgânicos. Revista Verde de Agroecologia e Desenvolvimento Sustentável, 13(2), 265-269. http://dx.doi.org/10.18378/rvads.v13i2.5632

Quiroz, M., \& Céspedes, C. (2019). Bokashi as an Amendment and Source of Nitrogen in Sustainable Agricultural Systems: a Review. Journal of Soil Science and Plant Nutrition, 19(1), 237-248. https://doi.org/10.1007/s42729-019-0009-9

Rocha, H. F., Leonardo, F. V. S., \& Oliveira, A. C. (2015). Plantios comerciais de Tectona grandis Lf no Brasil. Multitemas. http://dx.doi.org/10.20435 /multi.v0i48.137

Santos, C. C., Bernardes, R. D. S., Goelzer, A., Geist, M. L., Vieira, M. D. C., \& Zárate, N. A. H. (2019). Bokashi on seedlings of Campomanesia adamantium (Cambess.) O. Berg: morphometric and photochemical aspects. Nativa: Pesquisas Agrárias e Ambientais, 7(3), 239-243. http://dx.doi.org/10.31413/nativa.v7i3.6772

Siqueira, A. P. P., Siqueira, M. F. B. (2013). Bokashi: adubo orgânico fermentado. Programa Rio Rural. (Manual Técnico, 40).

Souza, C. A. M. D., Oliveira, R. B. D., Martins Filho, S., \& Lima, J. S. D. S. (2006). Crescimento em campo de espécies florestais em diferentes condições de adubações. Ciência Florestal, 16(3), 243-249. http://dx.doi.org/10.5902/198050981905

Souza, F. M., Lima, E. C. S., da Silva Sá, F. V., Souto, L. S., Araújo, J. E. S., \& de Paiva, E. P. (2017). Crescimento inicial do milho sob doses de esterco caprino e disponibilidade de água no solo. Revista Verde de Agroecologia e Desenvolvimento Sustentável, 12(2), 241-245. http://dx.doi.org/10.18378/rvads.v12i2.4964

Trazzi, P. A., Caldeira, M. V. W., Passos, R. R., \& Gonçalves, E. D. O. (2013). Substratos de origem orgânica para produção de mudas de teca (Tectona grandis Linn. F.). Ciência Florestal, 23(3), 401-409.

Trazzi, P. A., Caldeira, M. V. W., Reis, E. F. D., \& Silva, A. G. D. (2014). Produção de mudas de Tectona grandis em substratos formulados com biossólido. Cerne, 20(2), 293-302. https://doi.org/10.1590/01047760.201420021134

Wendling, I. (2002). Substratos, adubação e irrigação na produção de mudas. Aprenda Fácil. 